\title{
Estrategias de competitividad para Pymes del sector manufacturero en méxico
}

\author{
Victoria Hernández Ramírez * \\ Claudia Gómez Vital ${ }^{\star *}$
}

\section{RESUMEN}

El presente documento es producto de una investigación sobre estrategias de competitividad que se centra en la calidad del servicio al cliente, aplicado al sector de manufactura en México y tomando como caso de estudio la empresa Porcelanas Ánfora en el Estado de Hidalgo.

Los resultados obtenidos en las encuestas y cuestionarios aplicados a los principales clientes y al personal de Porcelanas Ánfora, compañía utilizada como referencia para este trabajo, confirman la hipótesis y comprueban que las empresas de manufactura pueden ser más competitivas si mejoran la calidad del servicio al cliente.

\section{INTRODUCCIÓN}

$\mathbf{E}$ estudio se presenta como una alternativa de solución a los empresarios, con el fin de que toda esta información les sea útil para analizar la situación actual de la empresa, que tengan los antecedentes, las bases y fundamentos aquí presentados para implantar un modelo de calidad de servi- cio al cliente que les permita mejorar su ventaja competitiva, incrementar sus ventas, conservar su imagen y posición en el mercado pero, sobre todo, mantener y mejorar su rentabilidad que es, a fin de cuentas, el verdadero objetivo de cualquier empresa.

\footnotetext{
* Directora de Investigación y Posgrado del Instituto Tecnológico Latinoamericano.

** Egresada de la Maestría en Mercadotecnia del Instituto Tecnológico Latinoamericano.
} 


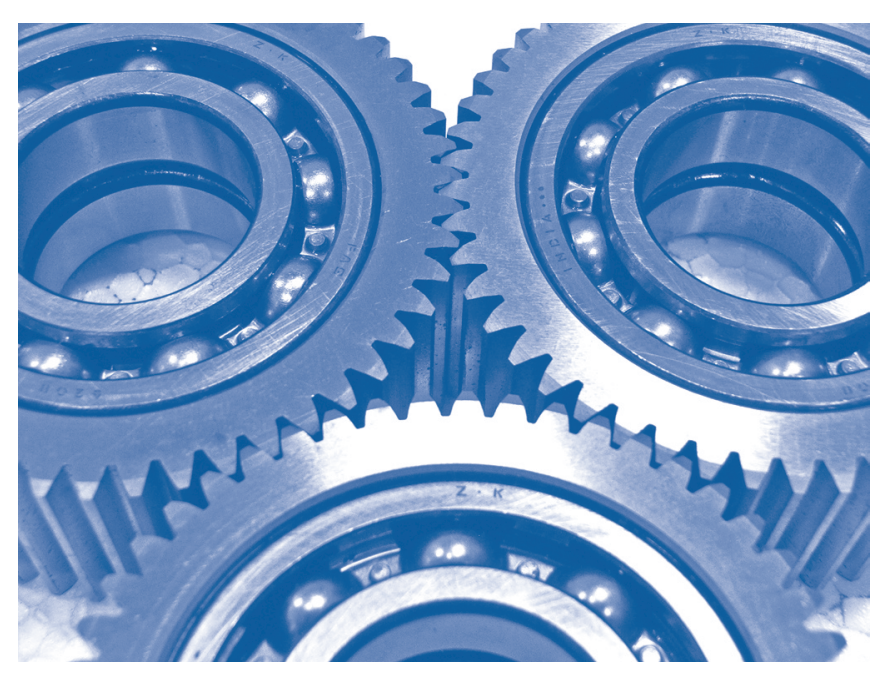

Metodología

Se eligió utilizar el enfoque mixto, en virtud de que es un proceso que recolecta, analiza y vincula datos cuantitativos y cualitativos en un mismo estudio para responder distintas preguntas de un planteamiento o problema (Teddlie y Tashakkori, 2003; Creswell, 2005; Mertens, 2005; Williams, Unrau y Grinnell, 2005).

Bajo esta perspectiva, este enfoque ha sido el apoyo ideal para hacer un análisis crítico, aplicando la lógica inductiva y deductiva al mismo tiempo, con el fin de comprender e interpretar los fenómenos que intervienen en el objeto de estudio, a través de las percepciones y significados producidos también de las experiencias de los participantes.

\section{Sustento teórico}

Hoy más que nunca las empresas, para subsistir, deberán ser más competitivas, lo que implica que habrán de apoyarse en las herramientas que les permitan lograr este objetivo y poder así permanecer en el mercado. Los índices de competitividad a escala Latinoaméricana que reporta el Foro Económico Mundial no son nada alentadores, especialmente cuando México del lugar 60 pasa al número 66 en el último reporte (World Economic Forum, 2010).
Sin duda, elevar la competitividad a escala país, requerirá que pequeñas y medianas empresas desarrollen y apliquen estrategias que les permita sumarse y, sobre todo, permanecer con éxito en los mercados. Por tanto, para lograr este importante objetivo, se requiere del estudio y el análisis de diversos factores como son los precios, la tecnología, la capacidad de innovación y la calidad, entendiendo este último concepto no solo desde el enfoque de producto, sino del de servicio, estrategia específica que se aborda en el presente documento.

Por tanto, en materia de competitividad, los empresarios del sector industrial han tenido que enfrentarse a auténticas pruebas de fuego. El reto que tienen ahora es todavía más fuerte y no porque deban superar nuevas exigencias técnicas o tecnológicas, sino porque los obliga a un cambio de mentalidad. Este cambio catártico debe producirse porque es la única manera de sintonizar con el cliente de hoy, el cual se mueve en el plano empiece a tener de verdad (Fernández, 2007). Por tanto, es un desafío para el sector industrial, y para quienes quieran permanecer en el mercado, entrar a un esquema de aprendizaje continuo que implica apertura y cambio de mentalidad en los ámbitos personal y organizacional para entrar a los procesos de competitividad.

Son las aportaciones de Porter las que en los últimos años van cobrando mayor influencia en el estudio de este tema. En este sentido, el término de competitividad se aplica en la actualidad a una empresa, a un sector económico o a un País. Porter (1991) establece que su significado puede ser diferente cuando se habla de una empresa, una nación o también de acuerdo con la especialidad o enfoque. Cuando se habla de una empresa se entiende como la habilidad para diseñar, producir y comercializar bienes y servicios, en donde habrá de distinguirse con un valor agregado más atractivo que la de sus competidores. Para un sector, la competitividad se entiende como la capacidad o potencial que tiene 
para crecer y contar con un rendimiento atractivo sobre la inversión realizada, en tanto para un país es la capacidad para competir en un mercado.

Desde enfoque nacional, la competitividad para Porter (1991) es ofrecer calidad y menor precio en la producción de bienes y servicios, beneficia a los habitantes de un país por tener un incremento en los ingresos reales. Por su parte, para Samuelson y Nordhaus (2003) la competitividad se refiere al grado en que pueden competir los bienes o servicios de un país.

Con un enfoque sectorial Hernández citado por Romo, (2008, pág. 58), refiere que la competitividad "es la capacidad de una industria o rama para no dejarse desplazar por importaciones, dentro de los mercados domésticos". Aspecto importante porque en la presente investigación, si bien se desarrolla en el estado de Hidalgo, participa un sector específico por demás afectado, como lo es el sector artesanal.

Desde la perspectiva de empresa, el concepto de competitividad tiene mayor coincidencia, de acuerdo con los diversos autores que la estudian, como son: Maidique y Patch (1978), Ortiz (1991), Carcoba (1991), Muller (1992), García (1993), Bueno (1995), citados por (Romo, 2008), donde refieren que es la capacidad que tiene una empresa para permanecer en el mercado, con algunas connotaciones que ya Porter y Deming toman al hablar de calidad y precio.

En tanto para Leonard D. Gonstein "la competitividad es la aplicación de la planeación estratégica para apagar incendios y manejar la crisis hacia una consideración proactiva del futuro y una forma de pensamiento dirigida a adelantarse a las jugadas del oponente" (Godstein, 1998).

La propuesta final del concepto de competitividad se presenta con una connotación sistémica, por tanto, es la capacidad de que una empresa, sector o país participe y esté posicio- nado en los mercados internos o internacionales y sea capaz de agregar valor a largo plazo a los productos o servicios que ofrezca (Hernández, (2010). En este sentido, el siguiente punto aborda el tema del sector manufacturero.

\section{Empresas del sector manufacturero en méxico}

La mayor proporción de la industria nacional se compone por empresas micro, pequeñas y medianas. Las grandes empresas representan proporcionalmente un número reducido.

Existen en el país aproximadamente tres millones y medio de empresas, donde laboran 17.1 millones de personas, de estas más del $40 \%$ son informales y se dedican a la venta de productos en las calles. La estructura empresarial mexicana se conforma principalmente por micro, pequeñas y medianas empresas. El 91,4\% del total de las unidades económicas y el 74,6\% del personal ocupado corresponden, en conjunto, a la industria manufacturera, al comercio y a los servicios (Gómez Ortíz, 2005).

La distribución en el territorio nacional muestra concentración diferente por tamaño. El 50\% de las microempresas se concentran en siete entidades de la zona centro del país. La empresa pequeña está en la zona centro y en el área metropolitana de la ciudad de México, mientras que la mediana está dispersa y la gran empresa se concentra en los estados norte del país. La distribución por sector se constituye con el $57 \%$ por empresas dedicadas al comercio, el 31,5\% a los servicios, $10,5 \%$ al sector manufacturero y el $1 \%$ a la industria de la construcción (Infopyme, 1998). Su principal ventaja es el uso intensivo de mano de obra al ocupar el 51\% del total del personal laborando en la industria.

"La actividad manufacturera constituye, después del sector comercial, el renglón de mayor peso dentro de la actividad económica del país, 23,6\% del total nacional, de ahí su im- 
portancia como determinante del crecimiento y, en su caso, de la contracción del PIB nacional" (Infopyme, 1998). Considerando que las empresas micro, pequeñas y medianas son las que mayor número de empleos generan y son más flexibles para adaptarse a las nuevas demandas que la sociedad genere, el sector manufacturero adquiere relevante importancia para el desarrollo social y económico del país. En este contexto los desafíos que enfrentan las empresas mexicanas son productividad, calidad, competitividad y cultura empresarial.

La empresa seleccionada para este trabajo se encuentra situada en el Estado de Hidalgo, en el municipio Mineral de la Reforma. Actualmente tiene laborando una plantilla de 350 empleados y tiene ventas anuales de más de 120 millones de pesos, por lo cual se podría clasificar como una mediana empresa.Sin embargo, derivado de que la industria de manufactura en el estado de Hidalgo no es tan sobresaliente como en los estados norte de la República, esta empresa es considerada una de las más grandes industrias de manufactura en el estado y la más grande del municipio Mineral de la Reforma.

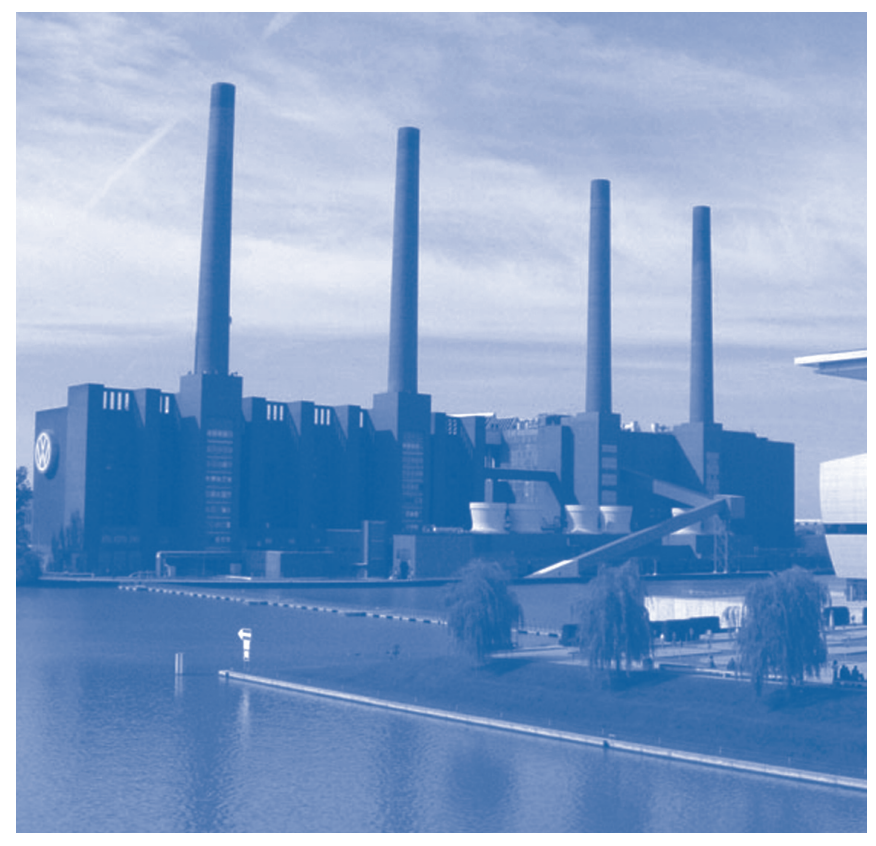

En el siguiente punto, se describen los resultados obtenidos, derivados de la aplicación de una entrevista guiada al personal directivo y gerencial que tiene contacto con los clientes, con el objeto de obtener información de aspectos relevantes de la empresa relacionados con la calidad en el servicio y atención al cliente, a fin de que sea la base para la toma de decisiones.

\section{Resultados obtenidos en la investigación}

\section{A) Calidad de servicio}

Antes de analizar los resultados obtenidos de esta categoría vale la pena reflexionar sobre lo que Deming, Jurán y Armand V. Feigenbaum (Larrea,1991) conciben de la calidad del servicio, la cual definen como "La satisfacción de las expectativas del cliente". Por tanto, se debe considerar lo complicada que puede ser la tarea de la calidad hoy, toda vez que es difícil precisar cuáles son las necesidades o requerimientos del mercado, dada la naturaleza subjetiva, cualitativa y cambiante que presentan los deseos del cliente. La calidad de los productos puede derivar de un buen trabajo del área investigación y desarrollo, pero la calidad de los servicios de la empresa da una idea más amplia de la experiencia que el cliente vive con la empresa, donde se involucra a todas y cada una de las áreas de la organización. Por lo que la investigación resalta la importancia de la calidad en los servicios como una estrategia alcanzable, y sin un alto costo para la organización, y sí un beneficio directo en los indicadores que miden la competitividad, como son las utilidades y las ventas.

En las respuestas obtenidas de las entrevistas, se detectó que las principales inconformidades que manifiestan los clientes en relación con la calidad de servicio son:

- La impuntualidad en los tiempos de entrega.

- No tener productos para entrega inmediata. 
- Incumplimiento de las promesas establecidas.

- Deshonestidad en la información que se le proporciona al cliente.

- Deficiencia en la coordinación logística en las entregas.

- Falta de atención personalizada por parte de los vendedores.

Estos resultados, reafirman los obtenidos en los cuestionarios, los cuales muestran que las expectativas de los clientes no son cubiertas de manera integral y permiten identificar cuáles son los puntos débiles.

Analizando lo anterior se detectan dos problemas principales: la impuntualidad en los tiempos de entrega y la falta de atención personalizada por parte de los vendedores. Los demás son factores negativos que se derivan de estos.

En este caso, el primero detona los demás factores negativos si se considera que no entregar en tiempo significa incumplir la promesa establecida. Algunos incumplimientos son originados por las fallas de logística en la entrega y el personal es deshonesto con el cliente porque prefiere decirle mentiras para no quedar mal, en vez de hablarle con la verdad.

Para solucionar este problema se recomienda hacer reajustes internos basados en un análisis exhaustivo sobre los resultados de producción en la planta, en cuanto a volumen, eficacia y eficiencia para detectar cuáles son las debilidades que se deben mejorar con el objetivo de contar con el producto requerido para cubrir entregas en tiempo y forma.

Las entregas oportunas dependen también de la eficiencia en la coordinación logística, por lo que se recomienda revisar paso a paso el proceso de entrega para verificar que cumple con el procedimiento establecido que debe seguir cada uno de los involucrados y detectar el origen de las fallas para solucionarlas.

Es necesario reorientar al personal hacia una cultura de servicio honesta que verdaderamente la empresa pueda cumplir, como dice Pedro Larrea, (1991) se debe ser cuidadoso con las promesas que se le hacen a los clientes y asegurarse de que se pueden cumplir. Cuando a un cliente se le cumplen por debajo de lo que espera sencillamente se decepciona y es muy difícil recuperar su confianza, más aún si se le dicen mentiras porque con esto no solo se pierde la venta, sino también se daña seriamente la imagen de la empresa.

Asimismo, es imprescindible establecer canales de comunicación frecuente con el cliente, basados en sus expectativas para no invertir en falso e implantar acciones que no son percibidas ni valoradas.

El segundo problema que se percibe es la falta de atención personalizada al cliente por parte de los vendedores, Considerando que la fuerza de ventas es el espíritu de la empresa, Kotler (2006) lo define muy acertadamente como el intermediario entre la empresa y el cliente. Entonces, el trabajo del vendedor está más cerca del cliente que de la empresa; de la calidad de servicio y buena relación que logre con los clientes depende en gran parte la mayoría de las ventas que pueda conseguir.

Theodore Levitt citado por Larrea (1991), menciona que "si la estrategia del pasado de cara a los clientes se centró en las ventas, ahora está centrada en el marketing y en un futuro corto estará centrada en las relaciones con los clientes". Se recomienda hacer un replanteamiento de los factores importantes que refuerzan la relación con los clientes en el pasado, en el presente y en el futuro y desarrollar estrategias para reorganizar la función del personal de ventas orientada a un contacto constante y personalizado con los clientes, solo de 
esta manera se puede estar pendiente de las necesidades de los clientes para brindarles la satisfacción esperada con base en sus expectativas.

\section{B) Atención al cliente}

El concepto que abarca el concepto de atención al cliente, consiste en los encuentros que los clientes tienen con los proveedores elegidos, esto durante la entrega y consumo del producto o servicio. ¿De qué manera interactúan los clientes con las instalaciones, el personal e incluso con otros clientes? ¿Cuáles son las expectativas en cada uno de los pasos de la entrega del producto o servicio?

El beneficio de la compañía es precisamente la consecuencia de la satisfacción de los clientes, sin ellos, simplemente el negocio no tendría razón de ser. Un negocio sin clientes fieles prácticamente es una empresa en agonía.

Pedro Larrea (1991) puntualiza que la satisfacción del cliente se entiende como el conocimiento que la empresa tiene de sus clientes, su sistema de servicio, la capacidad de respuesta y la aptitud para satisfacer los requerimientos y expectativas de los consumidores. Ahora bien, se debe determinar si la experiencia del uso del servicio cubre las expectativas de atención al cliente y si los deja satisfechos y listos para repetir su compra en el futuro.

Después de esta breve introducción que ayuda a comprender mejor la finalidad de atención al cliente, se mencionan las fallas detectadas a través de la entrevista, que son:

- Llamadas telefónicas no atendidas .

- No le llaman, ni le envían información sobre el estatus de sus pedidos.

- No le proporcionan precios ni información completa de
Ios productos cuando los solicita, tienen que esperar hasta que haya una persona de ventas disponible.

- Errores o extravíos de documentos que retrasan la captura y entregas.

- Quejas o inconformidades sin solución.

La función principal de atención al cliente, como su nombre lo indica, es asistirlo cuando lo requiera, proporcionándole información sobre las características, el uso, el precio y las ventajas de los productos, darle seguimiento e informarle sobre el estatus de sus pedidos, atender sus quejas. En fin, cualquier dato que soliciten o deseen saber relacionado con la compra o derivado de esta.

Para tal fin se les debe crear la cultura organizacional, a través del conocimiento de la información relacionada con la empresa y sus productos y mediante la formación constante sobre la cultura de servicio al cliente. Como reconoce Peter R. Heinze, director de la División Química de BASF, citado por Kotler (2006), los clientes necesitan algo más que un simple proveedor, necesitan una especie de socio, alguien que pueda proporcionar asesoramiento, información y productos de alta calidad.

Si se quiere que el personal sea eficiente, es importante brindarle las herramientas necesarias para llevar a cabo sus funciones de una forma más rápida y fácil, proporcionarle capacitación constante así como establecer un incentivo de estímulos y recompensas en proporción con el cumplimiento de los objetivos de cada departamento. Lo anterior, con el fin de fomentar el trabajo en equipo, mejorar la comunicación y propiciar un buen clima laborar basado en la confianza y el respeto, que refuerce la motivación del personal, la cual es factor determinante de la buena actitud, que es un aspecto indispensable que deben tener quienes tienen contacto con el cliente. 
El proporcionar una buena atención, implica conocer las expectativas del cliente hoy, mañana y dentro de cinco años. Lo de hoy es preocuparnos por la satisfacción del cliente, abrirle a cada cliente lo que es nuestra empresa y lo que tiene, para que él escoja en qué medida se le puede ayudar. Se trata de convertir al cliente en el elemento clave de la compañía a través del cumplimiento cabal de sus requerimientos.

\section{C) Competitividad}

Para comprender la importancia que representa la competitividad en las empresas hay que analizar su definición. Garza (2000) dice que competitividad es ofrecer calidad, precio y servicio en el ámbito de las mejores organizaciones, para lo cual es necesario establecer una estrategia, proponerse metas y conseguirlas, pues si no hay resultados de nada sirve.

La competitividad se relaciona con la excelencia y el cumplimiento de expectativas. Los resultados deben mostrar niveles de excelencia, satisfaciendo los requisitos deseados y, sobre todo, lograr superioridad sobre otras opciones existentes en el mercado.

Las afirmaciones anteriores dejan claro que el ser competitivo es simplemente ser mejor que la competencia y convertirse en "la mejor opción para el consumidor", lo cual implica brindar calidad, funcionalidad, innovación, precio, servicio, atención, ventajas y cualquier otro tipo de beneficio que nuestros productos o servicios le puedan ofrecer al cliente mejor que el de nuestros competidores y que cumplan con sus expectativas.

Para mejorar la competitividad de Ánfora, los entrevistados coincidieron en llevar a cabo una estrategia de reajustes internos que nos permita corregir las fallas detectadas en cuanto a calidad de servicio y atención al cliente.
Para solucionar el problema relacionado con los tiempos de entrega, es necesario contar con una producción eficiente, por lo que se recomienda hacer una reingeniería al área de producción, de tal forma que se haga una selección de productos viables basados en criterios de los más vendidos en cuanto a volumen y a la utilidad que representan para la empresa. Entonces proceder a descontinuar los que ya no representan beneficio o merman considerablemente la utilidad, pues más vale compactarse, producir menos y ganar, que perder por conservar tanta variedad de líneas y tipos de artículos.

Una de las garantías de Ánfora es la reposición de los productos. Sin embargo, la empresa puede determinar con qué líneas va a continuar trabajando para garantizar no solo la reposición de las piezas, sino también mayores beneficios al cliente, tales como tiempos de entrega más cortos, stock de artículos en blanco para entrega inmediata, más versatilidad en decorados, mejores precios, y como consecuencia, mayor calidad en el servicio, la cual es la más importante de las ventajas competitivas hoy en día.

Como lo infiere Michael Porter (2004), para llevar a cabo cualquier estrategia competitiva se deben considerar las fortalezas, debilidades, oportunidades y amenazas de la empresa y compararlas con las de la competencia. En definitiva, conocer las expectativas que los clientes tienen ante la oferta de los competidores, averiguar la percepción que sienten respecto a su desempeño real, detectar su estrategia actual y aventurar la futura, permiten en conjunto orientar con mayor racionalidad la política de servicio por seguir para lograr ser más competitivos que ellos.

Sin embargo, cualquier estrategia competitiva no tendrá éxito sin la estructura organizacional adecuada, pues se requiere la colaboración del personal con los atributos necesarios para conseguirlo. 
Los departamentos de atención al cliente y ventas son piezas clave de la estrategia competitiva de la empresa, por tanto, el área de relaciones industriales debe asegurarse con base en una buena planeación, manual de procedimientos y descripción de puestos, que la estructura de esas áreas este acorde con el giro de la empresa y con el número de clientes que tiene.

En cuanto al reclutamiento, se recomienda seleccionar personal calificado, con experiencia y, sobre todo, con habilidades de negociación y comunicación asertiva, o de otro modo, formarlos mediante la capacitación constante, con la finalidad de establecer negociaciones inteligentes y duraderas que atraigan ventas constantes para sostener la rentabilidad de la empresa.

\section{CONCLUSIONES}

- Al final de este análisis se concluye que el implantar el proceso de calidad en el servicio es cuestión de cultura organizacional, actitud e involucramiento del personal y de compromiso y disciplina. Se manejan factores objetivos de forma y subjetivos de fondo. La calidad no es solo la coherencia entre las propiedades de un bien y las exigencias del cliente, es más bien la correspondencia entre la percepción de las propiedades de dicho bien y lo que de él se espera, es la comparación entre la percepción del desempeño y las expectativas del comprador.

- El objetivo primordial del personal de atención al cliente debe ser lograr que cada cliente que tenga contacto con ellos compre alguno de nuestros productos, pero a la vez que quede encantado con el servicio y atención recibida para ganar su lealtad y se convierta entonces en cliente recurrente.
El personal de servicio al cliente no debe perder de vista su objetivo, debe estar consciente de dónde inicia y termina el proceso para lograrlo. Así pues, no debe colgar el teléfono, cerrar un e-mail, ni despegarse del cliente hasta no haber logrado conquistarlo y sostenerlo como cliente frecuente, con ello se sostiene también la rentabilidad y estabilidad de la empresa.

- La calidad va de la mano con la competitividad y esta, a su vez, con la rentabilidad. En la actualidad ya se evalúan las pérdidas que genera la no calidad; se trata de pérdida de clientes, disminución de sus compras, retraso en los pagos, impagos definitivos, concesión de descuentos compensatorios, repetición del servicio, devolución de la mercancía o su abaratamiento o pérdida total.

- Es indispensable contar con un plan de mejora continua y establecer estrategias de concentración y diferenciación, de acuerdo con los cambios que demande el mercado, con el fin de sostener la ventaja competitiva, que es la única forma de asegurar la permanencia y liquidez de las empresas.

- Por último, es importante puntualizar que los empresarios no deben confiarse y quedarse estáticos. Hoy en día, a medida que la competencia y los costos aumentan, la calidad y la competitividad baja, se requiere de una mayor utilización del marketing de servicios. Las empresas enfrentan tres tareas principales de marketing: mejorar su diferenciación competitiva, la calidad de su servicio y su productividad. 


\section{REFERENCIAS}

- Alvarez-Torres, M. (1998). Manual de Competitividad: procedimientos y herramientas prácticas. México: Editorial Panorama.

- Aragon, A. y (2005). Factores asociados con el éxito competitivo de las Pymes industriales en España. Universia Business Review, 36-49.

- Ailes, R. (1998) Tú eres el mensaje: la comunicación a través de los gestos, la imagen y las palabras. Barcelona: Paidós.

- Alcaide, J.C. (2002). "Alta Fidelidad". México. Editorial ESIC. Prólogo.

- Babakus, E.; Yavas, U.; Karatepe, O.M. y Avci, T. (2003). The effect of management commitment to service quality on employees: Afective and performance outcomes. Journal of the academy of marketing, Science Vol. 31 No. 3 p.-272-286.

- Bagozzi, R. (1992). The self regulation of attitudes, intentions and Behaviour. Social Psicholohy Quarterly, Vol. 55 No. 2 pp 178-204.

- Barquero, J.D. (2002). Comunicación y Relaciones Públicas. Madrid: McGraw-Hill.

- Becvar, R.J. (1978). Métodos para la Comunicación Efectiva. México: Limusa.

- Benayas, E. (02 de abril de 2009). Altavista.com. El cliente, objeto del deseo. Recuperado el 23 de abril de 2009 en:http://webferret.search.com/click?wf6,+\%2BArt\%ED culos+\%2Bsobre+\%2BServicio+\%2Bal+\%2BCliente,,ow. ly\%2F1WQW, altavista,1.

- Berry, L. y Parasuraman, A. (1991) Marketing Services: Competing Through Quality. Nueva York: The Free Press.

- Bitner, M. y Zeithaml, V. (1996). Services Marketing. Nueva York: McGraw-Hill.

Blanco, A. (2007). Atención al Cliente. España: Pirámide, S.A.
- Brady, M. y Cronin, J. Jr (2001). Customer orientation effects on customer service perceptions and outcome behaviors. Journal of service research Vol. 3 No. 3, pp 241-251. doi: $10.1177 / 109467050133005$

- Brown, Stephen W., Evert Gummeson, Bo Edvardsson y Bengtove Gustavsson. (1991). Service Quality: Multidiciplinary and Munltinational Perspectives: Lexington, MA: Lexington Books.

- CARRACINTRA. (2008). Página Web de Canacintra. Recuperado el 30 de julio de 2009, de canacintra.org.mx: http://www. canacintra.org.mx/estructura/sectores_ind.html.

- Castañeda, L. (2006). El cliente es tu Patrón. México. Ediciones Poder.

- Colunga, C. (1995). La Calidad en el Servicio. México. Panorama Editorial.

- Cook, K. (2004). Guía Completa para el Marketing de la Pequeña y Mediana Empresa. México: Granica.

- D'Aprix, R. (1999). Comunicación para el Cambio: cómo conectar el lugar de trabajo con las cambiantes demandas del mercado. Barcelona: Granica.

- Deming, E. (1989). Calidad, Productividad y Competitividad, la Salida de la Crisis. Madrid, España: Ediciones Díaz de Santos.

- Díaz, P. (11 de febrero de 2006). Mi cliente y yo. El Servicio al cliente y sus beneficios. Recuperado el 25 de marzo de 2009 en: http://www.miclienteyyo.com.ar/el-servicio-al-cliente-ysus-beneficios.

- Dickson, F.J. (1974) El Éxito en la Administración de las Empresas Medianas y Pequeñas. México: Editorial Diana.

- Eyssautier, M. (2002) Metodología de la Investigación, Desarrollo de la Inteligencia. Colombia: Thompson Learning.

- Fernández, F. (1973) Dirección y Organización de Empresas Privadas y Públicas. Buenos Aires: Editorial Macchi. 
- Ferrel, O.C. y Michael, D., (2006). Fundamentos de Marketing. México. Pearson Learning Editores.

- Fischer, L. y Espejo, J. (2004). Mercadotecnia. México. McGraw Hill.

- Forrester, R. (2000). Empowerment rejuvenating a potent idea. Academy of management executive vol. 14 No. 3 PP 6780.

- Frederick, E. Webster, Jr. (2002). The Role of Marketing and the Firm: Londres: Sage Publications.

- Garza, J.G. (2000). Administración Contemporánea. México: McGraw Hill Interamericana.

- Michaelson, Gerald, y Michaelson, Ste. (2004). Estrategias de Marketing. España: McGraw Hill Interamericana.

- Gobierno de Uruguay (20 de diciembre de 2006). Ministerio de Economía y Finanzas, República Oriental de Uruguay. Desarrollo del sector privado. Principales indicadores para medir la competitividad. Recuperado el 20 de julio de 2009 en: www.mef.gub.uy/inversor/doc_04_indicadores_de_competitividad.pdf.

- Godstein, L.; Thimothy, N. y Pfeifer, W. (1998). Planeación Estratégica Aplicada. Colombia: McGraw Hill.

- Godstein, L. (1998). Planeación Estratégica Aplicada. Colombia: McGraw-Hill.

- Goldhaber, G. (1990). Comunicación Efectiva. México: Editorial Diana.

- Gómez, R.A. (2005). Perfil deseable del empresario mexicano para la innovación y el desarrollo tecnológico. México: Instituto de Investigaciones Económicas y Empresariales.

- Group, Boston Consulting (18 de octubre de 2007). Portal Web Lycos. Matrices para medir competitividad. Recuperado el 17 de julio de 2009 en: Usuarios.lycos.es/sextuplegrama/Matriz. html.

- Gummerson, E. (1993) Quality Management in Service Organizations. Nueva York: International Service Quality Association.
- Guns, B. (1996). Aprendizaje Organizacional, Cómo Ganar y Mantener la Competitividad. México: Prentice Hall Hispanoamericana.

- Lovelock, C.(1997). Mercadotecnia de Servicios. México: Prentice Hall Hispanoamericana.

- Hamish, P. y Thompson, M. (1999). How Cause Related Marketing Builds Brands. New York: John Wiley \& Sons Editions.

- Hartline, M, F. O. (1996). The management of customer contact service employees. An empirical investigation. Journal of Marketing, Vol 52, pp. $52-70$.

- Heskett, J. (1986). Managing in the Service Economy. Boston: Harvard Bussiness School Press.

- Hitt, M. (2004). Administración Estratégica. México: Thompson Editores.

- Horovitz, J. (2006). Los secretos del servicio al cliente, movimientos de la dirección para obtener resultados con los clientes. Madrid, España: Pearson Education.

- Huerta, J.J. y Rodríguez, G. (2006). Desarrollo de Habilidades Directivas. México: Pearson Education.

- Infopyme, (1998, Art. 1, octubre). Una visión general en el sector manufacturero. Noticias sobre la pequeña y mediana empresa en América Latina, pp. 1-7.

- Jirón Santandreu, Andrés. Gerente General de Gestcom. Blog en internet.Gestcom. Comunicación organizacional. Más allá del servicio al cliente, 18 de agosto del 2008, recuperado el 4 de agosto del 2009 en: Hyperlink http://www.gestcom.cl/tendencias/10-servicio-al-cliente. html.

- Kostecki, M. (1994) Marketing Strategies for Services. Oxford: Pergamon Press.

- Kotler P. y Armsgtrong, G. (2003). Fundamentos de Marketing. México. Pearson Educación.

- Kotle,r P. y Keller, K.L. (2006). Dirección de Marketing. México. Pearson Educación. 
- Kotler, P. y Lee, N. (2005). Corporate Social Responsibility Doing the Most Good for your Company and your Cause. Nueva York: John Wiley Editions

- Kotler, P. (1997). Marketing Management Analisis, Planning and Control. México: Prentice Hall.

- Kottler P. y Clarke, R. (1987) Marketing for Health Care Organizations. Englewood Cliffs, NJ: Prentice Hall.

- Langeard, E.; Bateson, J.; Lovelock, C. y Eiglier, P. (1981) Services Marketing: New Insights from Consumers and Managers. Cambridge, MA: Marketing Science Institute.

- Larrea, P. (1991). Calidad de Servicio, del Marketing a la Estrategia. Madrid España: Ediciones Díaz de Santos.

- Lovelock, C. y Wirtz, J. (2009) Marketing de Servicios. Personal Tecnología y Estrategia. México. Pearson Educación.

- Magazine, Estrategia (06 de Febrero de 2008). Gestiopolis.com. de Satisfacción y servicio al cliente. Recuperado el 12 de marzo de 2009 en: www.gestiopolis.com/administracion-estrategia/estrategia/calidad-en-la-atencion-al-cliente-1.htm.

- Martínez de Velazco, A. y Nosnik, A. (1988). Comunicación organizacional Práctica. México: Editorial Trillas.

- Montaño, A. (2005). Mercadotecnia, investigación y análisis para el éxito. México: Trillas.

- Morales, G. Y. (2000). Competitividad y estrategia: el enfoque de las competencias esenciales y el enfoque basado en los recursos. Contaduría y Administración, 47-63

- Münch, L. y García, J. (1997). Fundamentos de Administración. México: Trillas.

- Münch, L. y Ángeles, E. (2007) Métodos y Técnicas de Investigación. México: Editorial Trillas.

- Pineda, M. (2008, Año 14, No. 153, marzo). Estrategia para la calidad. Revista Manufactura, No. 12.
- Porter, E. M. (2004). Técnicas para el Análisis de los Sectores Industriales y de la Competencia. México: Compañía Editorial Continental.

- Rodríguez, J. (2000). Administración con Enfoque Estratégico. México: Editorial Trillas.

- Rodríguez, J. (2007). Administración de Pequeñas y Medianas Empresas. México: Thompson Editores.

- Hernández, R.; Baptista, P. y Fernández, C. (2008). Metodología de la Investigación. México. McGraw Hill.

- Oster, M. (2000). Análisis Moderno de la Competitividad. México: Oxford University Press México.

- Rivas-Tovar, L. A. (2006). ¿Cómo hacer una tesis de maestría? México: Ediciones Taller Abierto.

- Taylor, S.J. y Bogdan, R. (1987) Introducción a los Métodos Cualitativos de Investigación. Barcelona España: Ediciones Paidos Ibérica, S.A.

- Tscholl, J. (2007). Como Conservar Clientes con un Buen Servicio. México: Pax México Librería.

- Tscholl, J. (1994). Alcanzando la Excelencia Mediante el Servicio a los Clientes. España: Díaz de Santos.

- Valero C. Ariel, licenciado en Comercio Internacional, instructor y conferencista internacional. Director de Education Training y Consulting. Blog en internet.Enfasis.com. ¿Cómo lograr un mejor servicio al cliente? El cliente es un factor de competitividad y de convivencia. 31 de julio del 2009, recuperado el 4 de agosto del 2009 en: Hyperlink http://www.logistica.enfasis. com/notas/13849-como-lograr-un-mejor-servicio-al-cliente.

- Villareal, R. (17 de agosto de 2004). México hacia una economía avanzada en el siglo XXI: Las condiciones para sus empresas. La competitividad sistemica de México y el desarrollo sustentable de sus Pymes: Recuperado el 16 de julio de 2009 en: www.google.com.mx/search?hl=es\&rlz=1W1ADBF_es\&ei

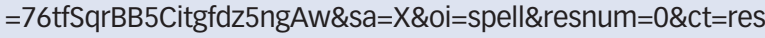
ult\&cd=1\&q=Rene+Villarreal\&spell=1.www.mef.gub.uy/inversor/doc_04_indicadores_de_competitividad.pdf . 